\title{
Medidas morfométricas del huevo fértil de codorniz (Coturnix coturnix japonica) sobre el peso al nacimiento
}

\author{
Morphometric measurements of the fertile quail egg (Coturnix coturnix japonica) \\ on chick weight at one-day old
}

\author{
Jimny Nuñez D. ${ }^{1,7}$, Nadia Fuentes N. ${ }^{2}$, Graciela Yamada A. ${ }^{2}$, Víctor Bazán R. ${ }^{2}$, \\ Steven Antúnez A. ${ }^{1}$, Virginia Rivadeneira ${ }^{3}$, Fritz Trillo Z. ${ }^{4}$, Pedro Ciriaco C. ${ }^{4}$, \\ Marco Gutierrez T. ${ }^{5}$, Connie Gallardo . $^{6}$
}

\section{Resumen}

\begin{abstract}
El estudio tuvo como objetivo relacionar medidas morfométricas del huevo de codorniz Coturnix coturnix japonica con el peso al nacimiento en una línea comercial de postura. Se evaluaron 274 aves al nacimiento, cuyos huevos fértiles fueron clasificados en tres categorías según el peso: (1) 10.00-10.99, (2) 11.00-11.99, (3) 12.00-12.99 g para la variable peso al nacimiento, mientras que las variables: diámetro transversal y diámetro longitudinal del huevo, índice de forma fueron evaluadas según cuartiles. Además se evaluó el sexo del ave. Se utilizó un modelo de bloques completamente al azar y correla-
\end{abstract}

${ }^{1}$ Laboratorio de Producción Avícola y Especies Menores, Facultad de Medicina Veterinaria, Universidad Nacional Mayor de San Marcos, Lima, Perú

${ }^{2}$ Centro de Investigación IVITA - Huaral, Facultad de Medicina Veterinaria, Universidad Nacional Mayor de San Marcos, Lima, Perú

${ }^{3}$ Laboratorio de Zootecnia y Producción Agropecuaria, Facultad de Medicina Veterinaria. Universidad Nacional Mayor de San Marcos, Lima, Perú

${ }^{4}$ Departamento de Producción Animal, Facultad de Zootecnia, Universidad Nacional Agraria La Molina, Lima, Perú

${ }^{5}$ Doctorado en Ciencia Animal, Facultad de Zootecnia, Universidad Nacional Agraria La Molina, Lima, Perú

${ }^{6}$ Departamento de Medicina Veterinaria y Ciencia Animal, Universidad Científica del Sur, Lima, Perú

${ }^{7}$ E-mail:jnunezd@unmsm.edu.pe

Recibido: 5 de abril de 2021

Aceptado para publicación: 9 de octubre de 2021

Publicado: 22 de diciembre de 2021

CLos autores. Este artículo es publicado por la Rev Inv Vet Perú de la Facultad de Medicina Veterinaria, Universidad Nacional Mayor de San Marcos. Este es un artículo de acceso abierto, distribuido bajo los términos de la licencia Creative Commons Atribución 4.0 Internacional (CC BY 4.0) [https:// creativecommons.org/licenses/by/4.0/deed.es] que permite el uso, distribución y reproducción en cualquier medio, siempre que la obra original sea debidamente citada de su fuente original 
ciones entre las variables cuantitativas. Se obtuvo una correlación media de 0.65 entre peso del huevo y diámetro longitudinal, y correlaciones bajas entre las variables diámetro longitudinal y transversal ( 0.15$)$. El peso al nacimiento fue de $7.36 \pm 0.038,7.92 \pm 0.036 \mathrm{y}$ $8.47 \pm 0.058 \mathrm{~g}$ en las categorías 1,2 y 3 , respectivamente y de $7.83 \pm 0.0672,7.81 \pm 0.0681$, $7.69 \pm 0.0665$ y $7.85 \pm 0.0682 \mathrm{~g}$ en los cuartiles $1,2,3$ y 4 , respectivamente. El índice de forma del huevo fue $70.22 \pm 0.321 \%$. El peso de las codornices macho fue $7.84 \pm 0.0347 \mathrm{y}$ en hembras de $8.00 \pm 0.0358(\mathrm{p}<0.05)$. El diámetro transversal y longitudinal del huevo permite seleccionar visualmente un huevo incubable, siendo el diámetro transversal el más preciso, debido a que no se quieren codornices pesadas al nacimiento.

Palabras clave: diámetro del huevo, índice de forma, peso al nacimiento, postura, sexo

\section{AbSTRACT}

The aim of this study was to relate morphometric measurements of the Coturnix coturnix japonica quail egg with the weight at birth in a commercial line of laying. In total, 274 birds were evaluated at one-day old, whose fertile eggs were classified into three categories according to weight: (1) 10.00-10.99, (2) 11.00-11.99, (3) 12.00-12.99 g for the variable birth weight, while the variables: transverse diameter and longitudinal diameter of the egg, and shape index were evaluated according to quartiles. In addition, the sex of the bird was evaluated. A completely randomized block model and correlations between the quantitative variables were used. A mean correlation of 0.65 was obtained between egg weight and longitudinal diameter, and low correlations between the variables longitudinal and transverse diameter (0.15). The birth weight was $7.36 \pm 0.038,7.92 \pm 0.036$ and $8.47 \pm 0.058 \mathrm{~g}$ in categories 1,2 and 3, respectively, and $7.83 \pm 0.0672,7.81 \pm 0.0681$, $7.69 \pm 0.0665$ and $7.85 \pm 0.0682 \mathrm{~g}$ in quartiles $1,2,3$ and 4 , respectively. The egg shape index was $70.22 \pm 0.321 \%$. The weight of the male quail was $7.84 \pm 0.0347$ and in females it was $8.00 \pm 0.0358(\mathrm{p}<0.05)$. The transverse and longitudinal diameter of the egg makes it possible to visually select a hatching egg, the transverse diameter being the most precise, since heavy quail at birth is not required.

Key words: egg diameter, shape index, birth weight, posture, sex

\section{INTRODUCCIÓN}

En el Perú la producción de huevos de codorniz (Coturnix coturnix japonica) es una actividad creciente, pero no se tiene estadísticas oficiales de esta producción, ya que solo es tomado en cuenta la producción de huevos de gallinas. Sin embargo, esta producción representa una contribución significativa a la economía familiar y seguridad alimentaria en países en vías de desarrollo (Talukder et al., 2020).
Las codornices debido a sus características de corto intervalo generacional (17 días de incubación), pronta madurez sexual e inicio de postura (42 días), es utilizado como adecuado modelo animal para experimentación (Hanusova et al., 2016), con una producción entre 250 y 300 huevos por campaña (Dahouda et al., 2013). La codorniz japonesa es un ave con dimorfismo sexual, cuyas hembras tienen un tamaño corporal mayor que los machos, a diferencia de otras especies de aves de corral (Ayoola et al., 2014). 
La fertilidad y la calidad del huevo influyen en la incubabilidad y el peso de la codorniz de un día (Farooq et al., 2001).

El peso promedio de las codornices japonesas al nacimiento es $8.95 \mathrm{~g}$, pudiendo alcanzar pesos de hasta $12.7 \mathrm{~g}$ (Barbieri et al., 2015); sin embargo, cuando se hace selección en líneas comerciales, se pueden alcanzar pesos promedio de $9.3 \pm 0.1 \mathrm{~g}$ (Vali, 2008). El diámetro longitudinal y transversal son buenos predictores del peso del huevo; además, el peso de la codorniz al nacimiento está relacionado con el diámetro longitudinal, peso del huevo e índice de forma (Farooq et al., 2003). El objetivo de esta investigación fue relacionar medidas morfométricas del huevo de codorniz con el peso al nacimiento en una línea comercial de postura cuyo sexo al nacimiento está ligado al color del plumaje.

\section{Materiales y Métodos}

El experimento se realizó en la sala de incubación del Laboratorio de Producción Avícola y Especies Menores de la Facultad de Medicina Veterinaria de la Universidad Nacional Mayor de San Marcos, Lima, Perú.

Se evaluaron 274 aves de una línea comercial de postura de codornices japonesas del tercio medio de postura, provenientes de una granja comercial donde se manejan cruzamientos para obtener aves cuyo sexo está ligado al color de plumaje. Los huevos fueron colectados en la mañana, se colocaron en bandejas para transporte y se trasladaron a la sala de incubación para la selección, clasificación (liviano: 10.00-10.99 g, mediano: 11.00-11.99 g, pesado: $12.00-12.99 \mathrm{~g}$ ) y desinfección. Para la incubación se utilizó la incubadora Masalles ${ }^{\circledR}$ con una capacidad de 9000 huevos. Los parámetros de incubación fueron: $37.5^{\circ} \mathrm{C}$ temperatura, $60 \%$ humedad relativa, y 1 volteo por hora. A los 15 días del inicio de la incubación, los huevos fueron trasladados a la nacedora (Granjero Técnico

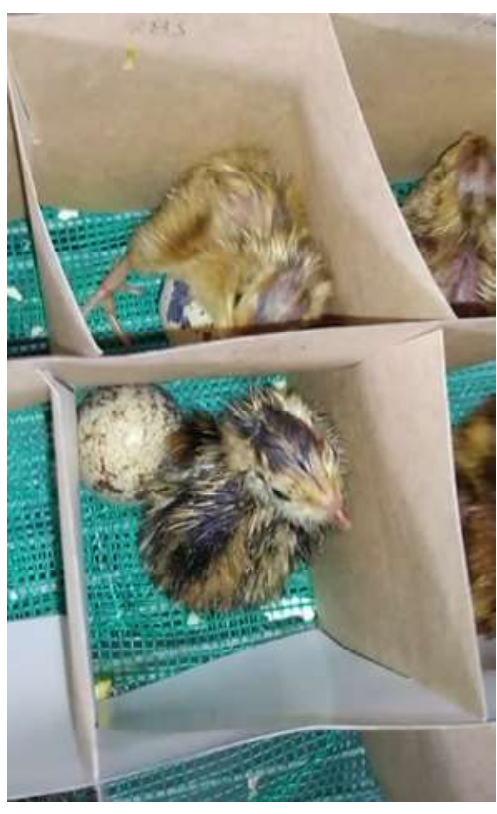

Figura 1. Sexo de la codorniz al nacimiento (superior: hembra, inferior: macho)

EIRL $^{\circledR}$ ) donde se mantuvo una temperatura de $37{ }^{\circ} \mathrm{C}$ y $75 \%$ de humedad relativa. El sexado se realizó en función a las características fenotípicas del animal, en este caso la coloración de plumaje más oscura indica el sexo macho (Figura 1).

Se tomaron medidas morfométricas (diámetro longitudinal [alto] y transversal [ancho]) del huevo utilizando un vernier digital de fibra de carbono Hedue ${ }^{\circledR}$ con una capacidad de $15 \mathrm{~cm}$ y una precisión de $0.1 \mathrm{~mm}$. Los huevos se pesaron en una balanza electrónica digital STMmax ${ }^{\circledR}$ de precisión $1 \mathrm{mg}$. El índice de forma fue calculado por el método de Anderson et al. (2004): (máximo diámetro transversal/diámetro longitudinal) x 100 . Las variables diámetro longitudinal y diámetro transversal del huevo y las categorías fueron analizadas con base a sus cuartiles.

El análisis estadístico se realizó mediante el modelo de bloques completamente al azar, donde el factor a evaluar fue la categoría por 
peso del huevo, sexo, cuartiles de índice de forma, y diámetro menor y mayor, restringidos por sexo. La variable evaluada fue peso al nacimiento de la codorniz. Las diferencias entre medias fueron determinadas mediante la prueba pos hoc de Tukey (alfa $=0.05$ ). Se utilizó el software R 4.04 con las librerías agricolae, lsmeans, multcomp y ggplot 2 .

\section{Resultados Y Discusión}

\section{Características Morfométricas}

El peso de huevo es un criterio importante en la clasificación de estos para la incubación. En el presente estudio se evidenció una asociación alta entre peso y diámetro longitudinal (0.65), mientras la asociación con el diámetro transversal fue media (0.42). Por otro lado, las variables diámetro longitudinal y transversal evidenciaron una baja asociación (0.15) (Figura 2).

Estas correlaciones fueron superiores a las registrados por Olawumi y Christiana (2017), quienes reportan valores de 0.51 (peso del huevo $v s$ diámetro longitudinal) y 0.28 (peso del huevo vs diámetro transversal). Por su parte, Aryee et al. (2020), al comparar el peso del huevo por encima de $11 \mathrm{~g}$ con el diámetro longitudinal, obtienen correlaciones positivas $(\mathrm{p}<0.05)$. Toda vez que el diámetro longitudinal y transversal tienen una baja correlación se recomendaría usar una razón altura diámetro para un análisis de la calidad del huevo.

\section{Categorías por Peso}

Los pesos promedio al nacimiento según la categoría del huevo de donde provinieron se muestra en el Cuadro 1, habiendo diferencias significativas entre las categorías (Figura 3). El menor límite de confianza se observó en la categoría liviana, en tanto que el mayor límite de confianza se presentó en la categoría de huevo pesado (Cuadro 1). Esto indica que posiblemente un mayor contenido

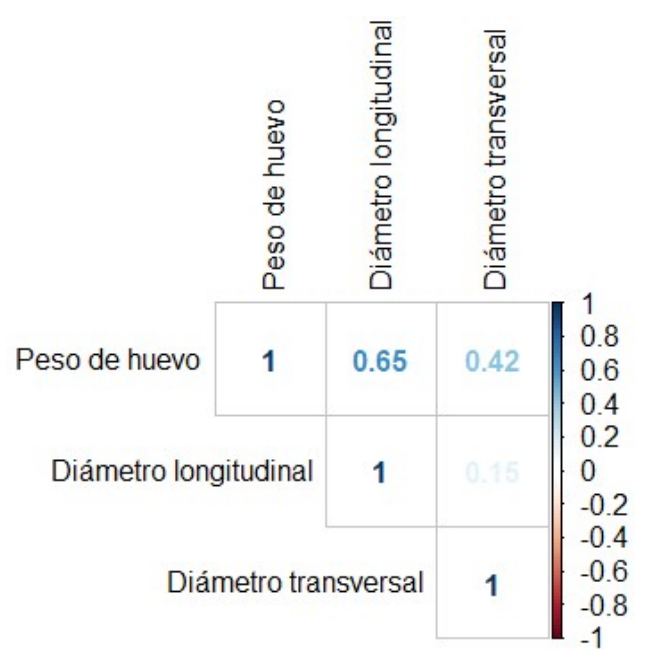

Figura 2. Correlación de las características morfométricas del huevo de codorniz

de yema y clara tienen influencia efectiva sobre el peso al nacimiento (Aryee et al., 2020). Asimismo, se conoce que la correlación entre el peso del huevo y peso al nacimiento es positiva (Ozcan et al., 2001). Por su parte, Gulsen et al. (2010) encontraron que huevos con pesos menores a $13.00 \mathrm{~g}$ resultan en aves de $8.41 \mathrm{~g}$ al nacimiento, en tanto que con huevos entre $13.01 \mathrm{y} 14.00 \mathrm{~g}$ se obtienen aves de $9.14 \mathrm{~g}$.

El peso del huevo de codorniz varia en base a línea de color Black $(9.95 \pm 0.88 \mathrm{~g})$; Brown $(10.53 \pm 1.06 \mathrm{~g})$ y White $(9.82 \pm 0.96$ g) (Chimezie et al., 2017) y línea liviana $(12.20 \pm 0.12 \mathrm{~g})$ y pesada $(13.26 \pm 0.10 \mathrm{~g})$ (Hanusova et al., 2016). Según esto, el peso del huevo reportado en este estudio estuvo por debajo de las líneas pesadas, pero coinciden con las líneas de color.

Caballero de la Calle et al. (1999), al evaluar huevos fértiles y peso de la codorniz europea al nacimiento, reportan que con huevos entre 9.5 y $10.0 \mathrm{~g}$ se obtienen codornices de $6.81 \pm 0.41 \mathrm{~g}$, además que a mayor peso del huevo mayor peso al nacimiento. En tan- 
Cuadro 1. Peso de la codorniz al nacimiento según el peso del huevo

\begin{tabular}{lcccccc}
\hline Categoría & $\begin{array}{c}\text { Peso del } \\
\text { huevo }(\mathrm{g})\end{array}$ & $\mathrm{n}$ & $\begin{array}{c}\text { Peso al } \\
\text { nacimiento } \\
(\mathrm{g})\end{array}$ & $\begin{array}{c}\text { Error } \\
\text { estándar }(\mathrm{g})\end{array}$ & $\begin{array}{c}\text { LC inferior } \\
(\mathrm{g})\end{array}$ & $\begin{array}{c}\text { LC superior } \\
(\mathrm{g})\end{array}$ \\
\hline 1 - Liviano & $10.00-10.99$ & 109 & 7.36 & 0.038 & 7.29 & 7.44 \\
2 - Mediano & $11.00-11.99$ & 119 & 7.92 & 0.036 & 7.85 & 7.99 \\
3 - Pesado & $12.00-12.99$ & 46 & 8.47 & 0.058 & 8.36 & 8.59 \\
\hline
\end{tabular}

LC: Limite de confianza

Cuadro 2. Diámetro longitudinal del huevo de codorniz por cuartil $(\mathrm{n}=274)$

\begin{tabular}{ccccccc}
\hline Cuartil & $\mathrm{n}$ & $\begin{array}{c}\text { Diámetro } \\
\text { longitudinal } \\
(\mathrm{mm})\end{array}$ & $\begin{array}{c}\text { Peso al } \\
\text { nacimiento } \\
(\mathrm{g})\end{array}$ & $\begin{array}{c}\text { Error } \\
\text { estándar }(\mathrm{g})\end{array}$ & $\begin{array}{c}\text { LC inferior } \\
(\mathrm{g})\end{array}$ & $\begin{array}{c}\text { LC superior } \\
(\mathrm{g})\end{array}$ \\
\hline 1 & 76 & $19.80-21.60$ & 7.49 & 0.065 & 7.36 & 7.62 \\
2 & 58 & $21.61-22.35$ & 7.61 & 0.057 & 7.50 & 7.72 \\
3 & 77 & $22.36-22.90$ & 7.83 & 0.062 & 7.71 & 7.96 \\
4 & 63 & $22.91-25.20$ & 8.17 & 0.057 & 8.06 & 8.28 \\
\hline
\end{tabular}

LC: Limite de Confianza

to, en el presente estudio el peso promedio al nacimiento fue de $7.36 \pm 0.038 \mathrm{~g}$ en huevos entre 10.00 y $10.99 \mathrm{~g}$ (Cuadro 1), siendo mayores a los de dicho reporte. Esto explica que el nivel de mejoramiento genético de las codornices Coturnix coturnix japonica ha ido en aumento.

\section{Diámetro Longitudinal}

El diámetro longitudinal de huevo en promedio fue $22.35 \pm 0.06 \mathrm{~mm}$. La distribución por diámetro longitudinal por cuartil se muestra en el Cuadro 2.

Al comparar las categorías de diámetro longitudinal del huevo de codorniz se evidenció diferencias estadísticas $(\mathrm{p}<0.01)$ en peso al nacimiento entre los cuartiles 3 y 4 , en tan- to que no hubo diferencias entre los cuartiles 1 y 2 (Figura 4). Esta comparación demuestra que el diámetro longitudinal del huevo de codorniz tiene influencia en el peso solo en cuartiles superiores ( 3 y 4 ).

Wilkanowska y Kokoszyñski (2012), al estudiar la codorniz Faraona (Coturnix coturnix Pharaon) de doble propósito, reportaron $32.6 \mathrm{~mm}$ como diámetro longitudinal de huevo, mientras que Hanusova et al. (2016) indican entre $33.00 \pm 10$ a $34.87 \pm 0.16 \mathrm{~mm}$ de diámetro longitudinal a los 220 y 175 días de postura, respectivamente, en la codorniz japonesa (Coturnix coturnix japonica). El diámetro longitudinal encontrado en esta investigación estuvo por debajo de los reportados por otros autores; sin embargo, al categorizarlos por cuartiles, se encontró una 


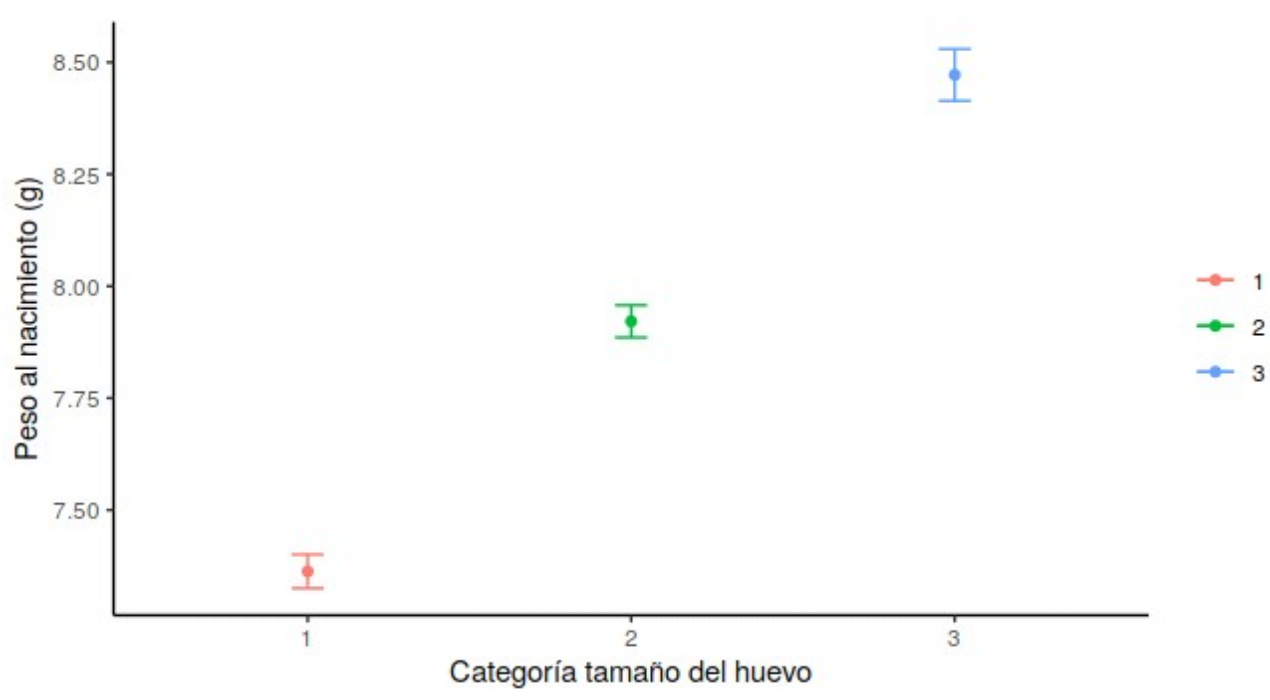

Figura 3. Comparación del peso al nacimiento $(\mathrm{g})$ de la codorniz con relación a la categoría del huevo (1: 10.00-10.99 g; 2: 11.00-11.99 g; 3: 12.00-12.99 g)

relación directa entre cuartil y peso de la codorniz al nacimiento.

\section{Diámetro Transversal}

El diámetro transversal promedio del huevo fue $15.67 \pm 0.07 \mathrm{~mm}$. La distribución por diámetro transversal por cuartil se muestra en el Cuadro 3.

No se encontraron diferencias significativas entre categorías de diámetro transversal del huevo de codorniz con relación al peso de la codorniz entre las categorías 2 y 3 , en tanto que los promedios de las categorías $1 \mathrm{y} 4$ fueron significativamente diferentes $(\mathrm{p}<0.01)$ entre sus promedios (Figura 5). Esta comparación indica que el diámetro transversal no tuvo influencia en las categorías intermedias; por lo tanto, solo los huevos de muy bajo diámetro transversal resultarían en codornices de bajo peso al nacimiento.
Como en el caso del diámetro longitudinal, Wilkanowska y Kokoszyñski (2012) reportaron un diámetro transversal promedio de $25.8 \mathrm{~mm}$ para la Coturnix coturnix Pharaon, en tanto que Hanusova et al. (2016) para la codornizjaponesa (Coturnix coturnix japonica) a los 175 días de edad presentó un diámetro transversal del huevo de $26.59 \pm 0.08 \mathrm{~mm}$, siendo similares a los reportados en este estudio (Cuadro 3). Por otro lado, Chimezie et al. (2017) al evaluar tres variedades de codornices japonesas reportaron $18.9 \pm 1.4,20 \pm 1.6$ y $19.1 \pm 1.1 \mathrm{~mm}$ de diámetro transversal en Black, Brown y White, respectivamente, valores que se encuentran dentro del rango del cuartil 4 en esta investigación.

\section{Índice de Forma del Huevo}

El promedio de índice de forma del huevo fue de $70.22 \pm 0.321 \%$. La distribución por cuartil se muestra en el Cuadro 4. No se 
Cuadro 3. Diámetro transversal del huevo de codorniz por cuartil $(\mathrm{n}=274)$

\begin{tabular}{ccccccc}
\hline Cuartil & $\mathrm{n}$ & $\begin{array}{c}\text { Diámetro } \\
\text { transversal } \\
(\mathrm{mm})\end{array}$ & $\begin{array}{c}\text { Peso al } \\
\text { nacimiento } \\
(\mathrm{g})\end{array}$ & $\begin{array}{c}\text { Error } \\
\text { estándar }(\mathrm{g})\end{array}$ & $\begin{array}{c}\text { LC inferior } \\
(\mathrm{g})\end{array}$ & $\begin{array}{c}\text { LC superior } \\
(\mathrm{g})\end{array}$ \\
\hline 1 & 86 & $11.50-15.20$ & 7.31 & 0.057 & 7.19 & 7.42 \\
2 & 65 & $15.21-15.60$ & 7.71 & 0.058 & 7.60 & 7.82 \\
3 & 58 & $15.61-16.00$ & 7.85 & 0.051 & 7.75 & 7.95 \\
4 & 65 & $16.01-26.20$ & 8.24 & 0.054 & 8.13 & 8.34 \\
\hline
\end{tabular}

LC: Limite de Confianza

Cuadro 4. Índice de forma de huevo de codorniz por cuartil $(\mathrm{n}=274)$

\begin{tabular}{ccccccc}
\hline Cuartil & $\mathrm{n}$ & $\begin{array}{c}\text { Índice de forma } \\
(\%)\end{array}$ & $\begin{array}{c}\text { Promedio } \\
(\mathrm{g})\end{array}$ & $\begin{array}{c}\text { Error } \\
\text { estándar }(\mathrm{g})\end{array}$ & $\begin{array}{c}\text { LC inferior } \\
(\mathrm{g})\end{array}$ & $\begin{array}{c}\text { LC superior } \\
(\mathrm{g})\end{array}$ \\
\hline 1 & 69 & $50.885-67.257$ & 7.83 & 0.0672 & 7.69 & 7.96 \\
2 & 68 & $67.257-69.773$ & 7.81 & 0.0681 & 7.68 & 7.94 \\
3 & 68 & $69.773-72.477$ & 7.69 & 0.0665 & 7.56 & 7.82 \\
4 & 69 & $72.477-113.914$ & 7.85 & 0.0682 & 7.72 & 7.99 \\
\hline
\end{tabular}

LC: Limite de Confianza

Cuadro 5. Peso al nacimiento según el sexo de la codorniz $(\mathrm{n}=274)$

\begin{tabular}{cccccc}
\hline Sexo & $\mathrm{n}$ & Promedio $(\mathrm{g})$ & $\begin{array}{c}\text { Error } \\
\text { estándar }(\mathrm{g})\end{array}$ & $\begin{array}{c}\text { LC inferior } \\
(\mathrm{g})\end{array}$ & $\begin{array}{c}\text { LC superior } \\
(\mathrm{g})\end{array}$ \\
\hline Macho & 146 & 7.84 & 0.0347 & 7.77 & 7.91 \\
Hembra & 128 & 8.00 & 0.0358 & 7.93 & 8.07 \\
\hline
\end{tabular}

LC: Limite de Confianza

evidenciaron diferencias significativas entre los promedios de los cuartiles a pesar de que se pudiese observar ligeras variaciones. Por otro lado, los pesos del cuartil 3 fueron los más bajos (Figura 6).
El índice de forma del huevo de codornices japonesas (Coturnix coturnix japonica) reportado para una línea denominada 8 (mayor peso vivo) fue de $76.451 \pm$ $0.388 \%$ y para una línea denominada 9 (con- 


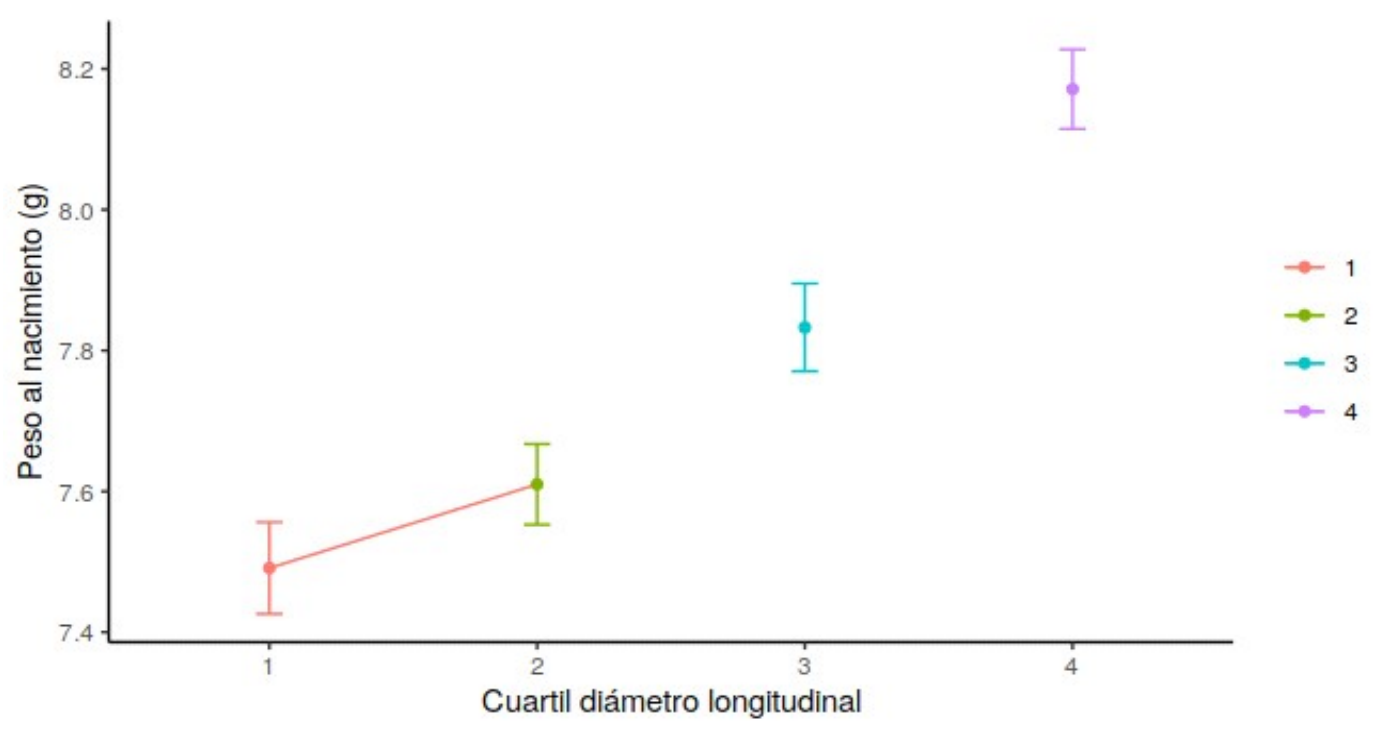

Figura 4. Comparación del peso vivo de la codorniz al nacimiento y cuartiles (diámetro longitudinal del huevo)

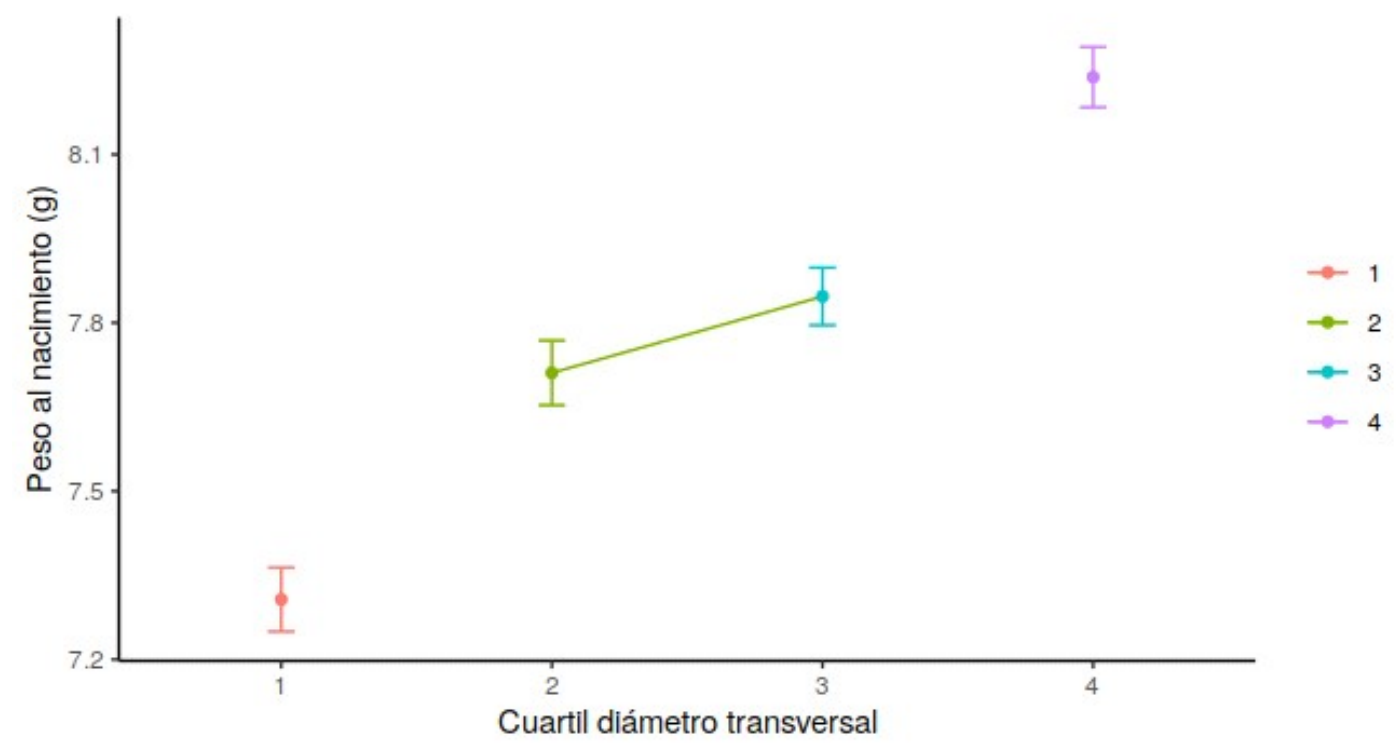

Figura 5. Comparación del peso vivo de la codorniz al nacimiento y cuartiles (diámetro transversal del huevo) 


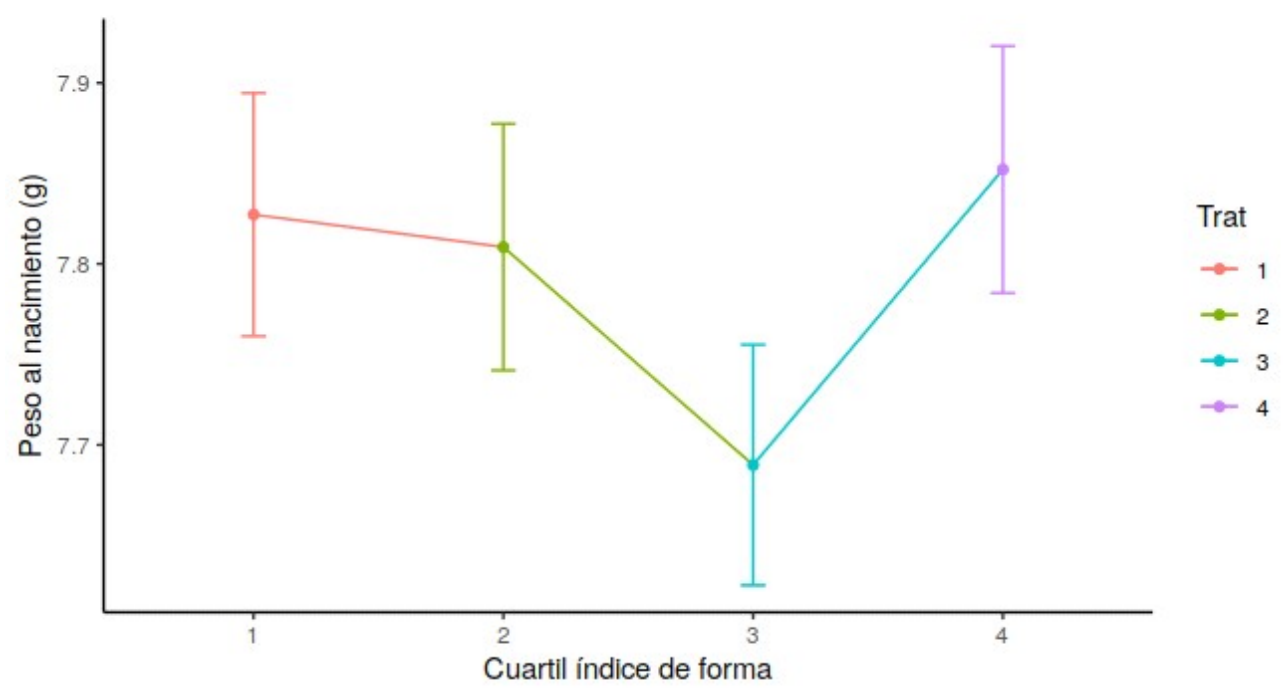

Figura 6. Comparación del peso de la codorniz al nacimiento del índice de forma del huevo por cuartiles

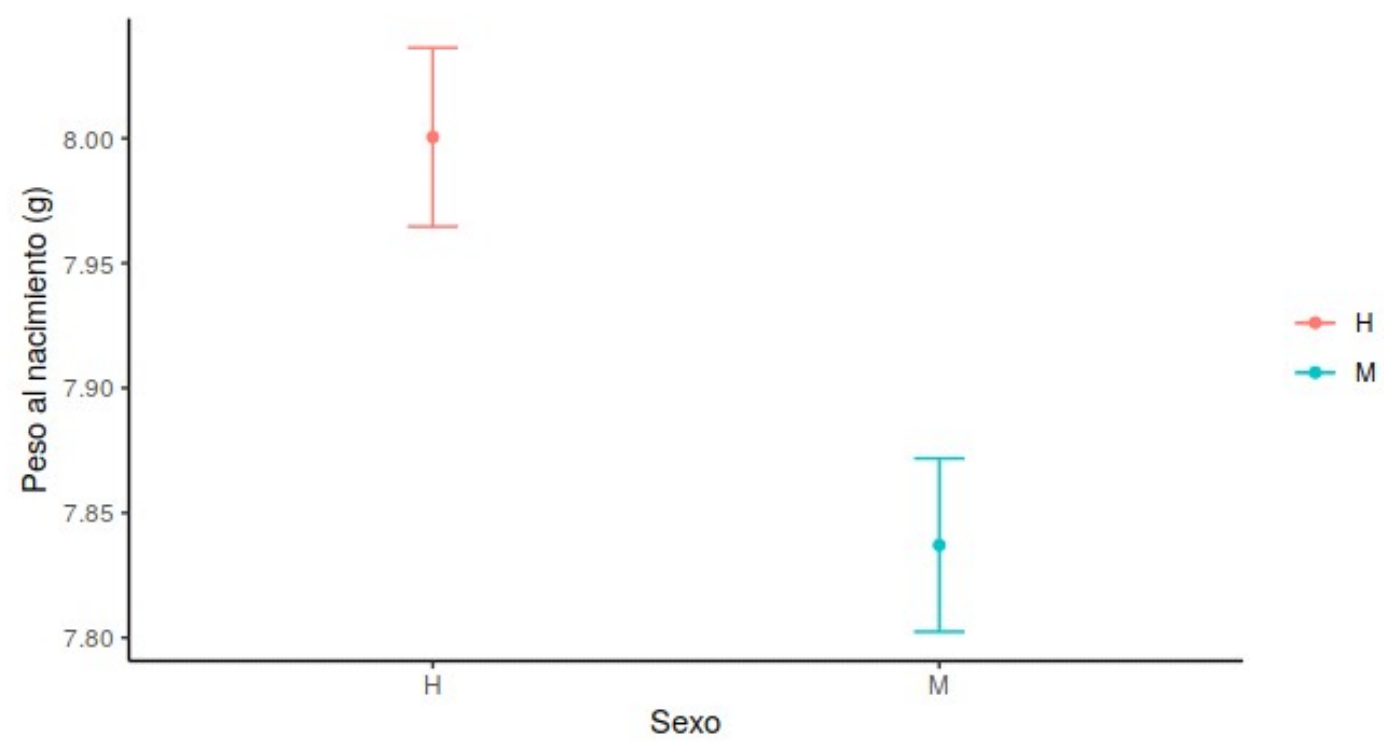

Figura 7. Comparación del peso de la codorniz al nacimiento según el sexo 
trol) fue de $78.575 \pm 0.420 \%$ (Hanusova et al., 2016); resultados que se encuentran dentro del rango del cuartil 4 en esta investigación. Por otro lado, se reportaron valores de $75.85 \pm 4.38,79.43 \pm 4.39$ y $76.12 \pm 5.92 \%$ en las variedades Black, Brown y White, respectivamente (Chimezie et al., 2017). Sin embargo, en esta investigación, el índice de forma de huevo en los tres primeros cuartiles tuvo valores inferiores a los reportados en la literatura, posiblemente debido a la línea comercial de postura evaluada.

El índice de forma reportado en el presente estudio $(113.913 \pm 0.0682 \%)$ demuestra que huevos con diámetro transversal mayor al longitudinal permiten obtener codornices de mayor peso al nacimiento; sin embargo, es posible que dichas aves no sean categorizadas como de buena calidad.

En codornices japonesas con índice de forma entre 70.00-73.85, 73.85-77.71 y 77.72$81.57 \%$ se reportaron pesos al nacimiento en hembras y machos de 9.23-8.99, 8.98-9.22 y 9.13-9.12 g, respectivamente, no habiendo diferencias significativas por sexo (Alasahan y Copur, 2016). Sin embargo, en el presente estudio se reportaron pesos menores al nacimiento.

\section{Efecto del Sexo en el Peso al Nacimiento}

Los pesos promedio por sexo al nacimiento se muestran en el Cuadro 5. Se observa una baja variabilidad dentro de sexo; sin embargo, el peso de las hembras fue significativamente mayor al de los machos $(\mathrm{p}<0.01$; Figura 7$)$. Esta diferencia a favor de las hembras posiblemente fue debida a la línea genética con tendencia a producción de huevos.

\section{Conclusiones}

- En líneas de codornices ponedoras Coturnix coturnix japonica, el diámetro transversal y longitudinal del huevo permite seleccionar visualmente un huevo incubable, siendo el diámetro transversal el más preciso.

- El peso al nacimiento de la codorniz estuvo influenciado por el sexo, siendo las hembras las que evidenciaron mayor peso.

\section{Literatura Citada}

1. Anderson KE, Tharrington JB, Curtis $P A$, Jones FT. 2004. Shell characteristics of eggs from historic strains of single comb White Leghorn chickens and the relationship of egg shape to shell strength. Rev Int Cienc Avícola 3: 17-19.

2. Alasahan S, Copur AG. 2016. Hatching characteristics and growth performance of eggs with different egg shapes. Braz J Poult Sci 18: 001-008. doi: 10.1590/1516-635x1801001-008

3. Aryee G, Adu AG, Ewusi SM, Nkrumah T, Amedorme D. 2020. Correlation between egg weight and egg characteristics in Japanese quail. Anim Vet Sci 8: 51. doi: 10.11648/j.avs.20200803.11

4. Ayoola, AA, Adeyemi OA, Egbeyale LT, Sogunle OM, Ekunseitan DA. 2014. Effects of sex and stocking density on growth performance and some physiological traits of Japanese quails (Coturnix coturnix japonica). Mal J Anim Sci 17: 43-53.

5. Barbieri A, Ono RK, Cursino LL, Farah MM, Pires MP, Bertipaglia TS, Fonseca R. 2015. Genetic parameters for body weight in meat quail. Poultry Sci 94: 169-171. doi: 10.3382/ps/peu062

6. Caballero de la Calle JR, Peña JC, Carrión E. 1999. Influencia de las características del huevo de la codorniz cinegética sobre la morfología del pollito. ITEA 20: 384-386.

7. Chimezie VO, Fayeye TR, Ayorinde KL, Adebunmi A. 2017. Phenotypic correlations between egg weight and some egg quality. J Agric Food Dev17: 44-53. 
8. Dahouda M, Adjolohoun S, Montchowui EH, Senou M, Hounsou MD, Amoussa S. 2013. Growth performance of quails (Coturnix coturnix) fed on diets containing either animal or vegetable protein sources. Int J Poult Sci 12: 396400. doi: 10.3923/ijps.2013.-396.400

9. Farooq M, Aneela K, Durrani FR, Muqarrab AK, Chand C, Khurshid A. 2001. Egg and shell weight, hatching and production performance of Japanese broiler quails. Sarhad J Agric 17: 289-293.

10. Farooq M, Durrani FR, Sarbiland K, Chand N. 2003. Predicting egg weight, shell weight, shell thickness and hatching chick weight of Japanese quails using various egg traits as regressors. Int $\mathrm{J}$ Poult Sci 2: 164-167. doi: 10.3923/ ijps.2003.164.167

11. Gulsen C, Mikail B, Sibel C, 2010. Egg weight but not egg shape index, determines the hatchability in Japanase quail (Coturnix coturnix japonica). J Anim Vet Adv 9: 1890-1895. doi: 10.3923/ javaa.2010.1890.1895

12. Hanusova E, Hrnèár C, Hanus A, Oravcová M. 2016. Egg traits in Japanese quails. Acta Fitotechn Zoo- techn 19: 62-67. doi: 10.15414/afz.2016.19.si.62-67

13. Olawumi S, Christiana B. 2017. Phenotypic correlations between external and internal egg quality traits of coturnix quails reared under intensive housing system. J Appl Life Sci Int 12: 1-6. doi: 10.9734/JALSI/2017/33802

14. Ozcan M, Ekiz B, Gunes H. 2001. Effects of egg weight and hatching weight size on growth performance in the Japanese quail (Coturnix coturnix japonica). Univ VetFact Derg 27: 577-584.

15. Talukder H, Sejuti DT, Jahid HT. 2020. Morphometric, productive and reproductive performances of Japanese quail (Coturnix japonica) in Sylhet city of Bangladesh. J Anim Breed Genet 4: 73-81. doi: 10.12972/jabng.20200007

16. Vali N. 2008. The Japanese quail: A review. Int J Poult Sci 7: 925-931. doi: 10.3923/ijps.2008.925.931

17. Wilkanowska A, Kokoszyñski D. 2012. Layer age and quality of Pharaoh quail eggs. J Central Eur Agric 13: 10-21. doi: 10.5513/JCEA01/13.1.1007 